\title{
Effective polymer concrete on waste concrete production
}

\author{
Evgeny Pyataev, Alexey Zhukov ${ }^{*}$, Kirill Vako, Marina Burtseva, Elizaveta Mednikova, \\ Maria Prusakova and Elizaveta Izumova \\ Moscow State University of Civil Engineering, Yaroslavskoe Shosse, 26, Moscow, 129337, Russia
}

\begin{abstract}
Finish materials in facade systems are experiencing significant stress associated with atmospheric and mechanical influences. The use of tiles with high durability and the ability to relax deformations will allow to obtain efficient cladding for building systems. The article presents the results of the selection of compositions and technologies of a composite material based on finely ground highly active waste produced by portland cement, mineral binder, additives based on polycarboxylic ether MC-6955, and polymer additive MC-Adhesive. MC-Adhesive is a polymer additive used for: a significant increase in flexural strength; reduce the elastic modulus; increase water resistance; increase the concrete mix cohesion; making coatings with high requirements for abrasion, low dusting and high resistance to aggressive substances. The article presents the results of research methods of selection of compositions of composite polymer concrete. The strength characteristics of polymer concrete with an average density of $2370-2450 \mathrm{~kg} / \mathrm{m}^{3}$ are most dependent on the consumption of fine waste and polymer additives. The nomogram obtained as a result of an active experiment makes it possible to evaluate the dependence of the strength on these factors and select their optimal ratio.
\end{abstract}

\section{Introduction}

The analysis of the information space united by the technology of polymer concrete and concretes on secondary raw materials showed that the sources of waste generation (including material debris) are demolition of construction sites and technological processes involving the accumulation of finely dispersed waste. As a result of the implementation of comprehensive housing programs, a huge amount of construction (including concrete) scrap has already accumulated at the respective storage facilities, the disposal of which is necessary, and will become mandatory in the near future.

There are many ways to utilize such by-products, but one of the science-based forms of waste disposal is to use them as part of building materials, including finishing materials [1, 2].

Note that by-products of technological processes can already become sources of fairly efficient raw materials or active fillers, especially since many of them initially possess binding properties $[3,4]$. The real way to recycle such waste is a using it as an active filler or binder component for building materials with special properties.

\footnotetext{
* Corresponding author: 1j211@yandex.ru
} 
Finishing materials in facade cladding systems have a significant atmospheric and mechanical load. Atmospheric loads are caused by temperature changes, direct action of condensed moisture, solar and wind effect. Mechanical loads are the result of changes in the geometric characteristics of the base (their shrinkage, movements, etc.), or with the deformations of the suspension and fastening systems. If the facing product is rigid, then upon reaching a certain level of stresses in the structure, it becomes possible to destroy the products or to drop them out of the fastening systems. For preserving the integrity of construction, it is necessary that the products have a certain elasticity (flexibility), while maintaining all performance characteristics at the level required by the standards $[3,4]$.

\section{Methods}

The goal of the research is the preparing and conduction an experiment aimed at creating the fundamentals of the technology of facing products based on mineral binder production wastes. The chemical composition of the waste was determined by the method of Fused Beads 12-05-14 on S8 TIGER (table 1).

Table 1. Chemical composition of waste.

\begin{tabular}{|c|c|c|c|c|c|c|c|c|c|}
\hline \multicolumn{1}{|c|}{ Chemical composition in the ignited state, \% } \\
\hline $\mathrm{SiO}_{2}$ & $\mathrm{Al}_{2} \mathrm{O}_{3}$ & $\mathrm{Fe}_{2} \mathrm{O}_{3}$ & $\mathrm{CaO}$ & $\mathrm{MgO}$ & $\mathrm{SO}_{3}$ & $\mathrm{~K}_{2} \mathrm{O}$ & $\mathrm{Na}_{2} \mathrm{O}$ & $\mathrm{MnO}$ & $\mathrm{TiO}_{2}$ \\
\hline 35.10 & 5.15 & 3.12 & 50.71 & 2.15 & 2.37 & 0.63 & 0.30 & 0.13 & 0.29 \\
\hline
\end{tabular}

The used cement in the studies - CEM II-42.5 (Heidelberg cement). As a superplasticizer in all compositions was used the additive based on polycarboxylic ether MC-6955 and a polymer additive MC-Adhesive. MC-Adhesive is a polymer additive used for: a significant increase in flexural strength; reduce the elastic modulus; increase water resistance; increase the concrete mix cohesion; making coatings with high requirements for abrasion, low dusting and high resistance to aggressive substances.

The experiment of the selection and optimization of the composition was carried out by the methods of mathematical planning of the experiment. The matrix of the experiment was based on a quadratic D-optimal rotary plan. The experimental conditions are given in table 2. Processing of the experimental results was carried out according to the method adopted for this method.

Table 2. The conditions of the experiment.

\begin{tabular}{|c|c|c|c|c|c|}
\hline Name of factor & $\begin{array}{c}\text { Symbol, } \\
\mathrm{X}_{\mathrm{i}}\end{array}$ & $\begin{array}{c}\text { The average } \\
\text { value of factor, } \\
\end{array}$ & $\overline{\mathrm{X}}_{i}$ & \multirow{2}{*}{$\begin{array}{c}\text { The range of } \\
\text { variation, } \\
\Delta \overline{\mathrm{X}}_{i}\end{array}$} & \multicolumn{2}{|c|}{$\begin{array}{c}\text { The values of } \\
\text { the factor levels }\end{array}$} \\
\cline { 5 - 7 } & & 70 & 10 & 60 & 80 \\
\hline $\begin{array}{c}\text { Disperse waste consumption, } \\
\mathrm{kg} / \mathrm{cm}^{3}\end{array}$ & $\mathrm{X}_{1}$ & & 0.2 & 0.8 & 1.2 \\
\hline Plasticizer consumption, \% & $\mathrm{X}_{2}$ & 1.0 & 0.4 & 4.6 & 5.4 \\
\hline $\begin{array}{c}\text { Polymer additive consumption, } \\
\%\end{array}$ & $\mathrm{X}_{3}$ & 5.0 & & & \\
\hline
\end{tabular}

The intervals of the change of factors were established on the basis of the analysis of a priori information and the goals of the experiment. The factors are independent and operable. The consumption of cement, sand, rubble and water in the experiment did not change. Cement consumption was assumed to be $264 \mathrm{~kg} / \mathrm{m}^{3}$, waste consumption $-66 \mathrm{~kg} / \mathrm{m}^{3}$ ( $20 \%$ of the initial cement mass), sand consumption - $900 \mathrm{~kg} / \mathrm{m}^{3}$, coarse aggregate consumption $1050 \mathrm{~kg} / \mathrm{m}^{3}$, water consumption $210 \mathrm{~kg} / \mathrm{m}^{3}$. 


\section{Results}

Mathematical processing of the experimental results allowed us to obtain regression equations for the average density $\left(\mathrm{U}_{2}\right)$ and compressive strength $\left(\mathrm{U}_{1}\right)$. The following mathematical models (polynomials) were obtained:

- for compressive strength:

$$
Y_{1}=32.6+2.8 X_{1}+1.9 X_{2}+1.8 X_{3}-1.4 X_{1} X_{3}
$$

Confidence interval for compressive strength $\Delta b_{1}=1.1 \mathrm{MPa}$.

- for medium density:

$$
Y_{2}=2415+21 X_{1}-14 X_{2}+12 X_{3}+10 X_{1} X_{3}+8 X_{2}^{2}
$$

The confidence interval for the average density is $\Delta b_{2}=7 \mathrm{~kg} / \mathrm{m}^{3}$.

The strength of polymer concrete samples is most affected by the consumption of finemilled waste (the coefficient at $\mathrm{X}_{1}$ is 2,8 ), and since this waste has the properties of a hydraulic binder, an increase in its consumption leads to an increase in compressive strength. The consumption of plasticizer and the consumption of polymer additives affect the increase in strength to a lesser extent (coefficients at $\mathrm{X}_{2}$ and $\mathrm{X}_{3}$ ). A simultaneous increase in the consumption of finely ground waste and polymer additives leads to a decrease in strength, which can be explained by a decrease in the reactive surface of the mineral component due to the adsorption of the polymer.

The average density of polymer concrete depends on variable factors to a lesser extent. The greatest impact on the average density has the consumption of fine ground waste (the coefficient at $\mathrm{X}_{1}$ is equal to 21). An increase in plasticizer consumption leads to a certain decrease in the average density, and this relation is not linear (the coefficients at $\mathrm{X}_{2}$ and $\mathrm{X}_{2}{ }^{2}$ are equal to " -14 " and 8 , respectively). An increase in the consumption of the polymer additive affects the increase in the average density to a lesser extent than other factors of the degree (the coefficient at $\mathrm{X}_{3}$ is equal to 12). A simultaneous increase (or decrease) in the consumption of the mineral component and the polymer additive slightly increases the average density (the coefficient at $\mathrm{X}_{1} \mathrm{X}_{3}$ is 10 ).

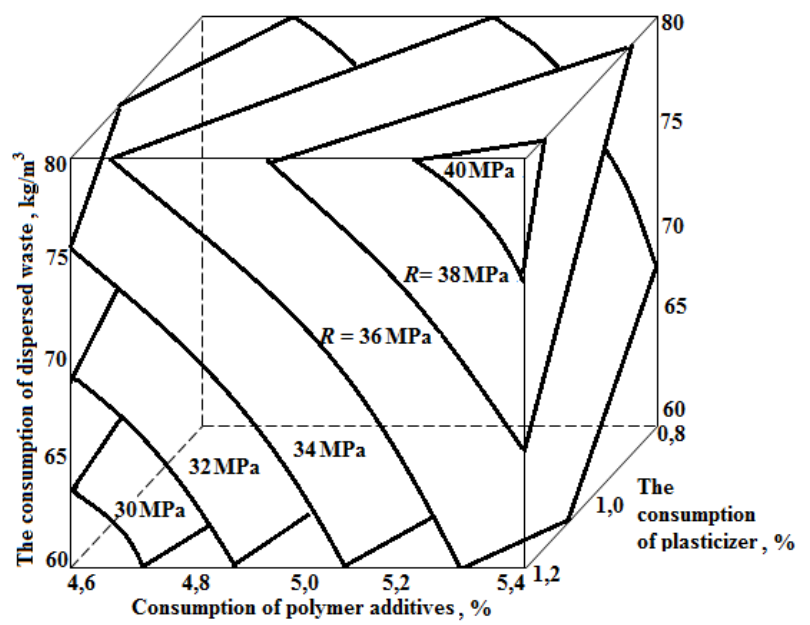

Fig. 1. Relation of compressive strength of polymer concrete samples (size 100x100x100 mm) on variable factors: dispersion waste costs, plasticizer and polymer additive. 


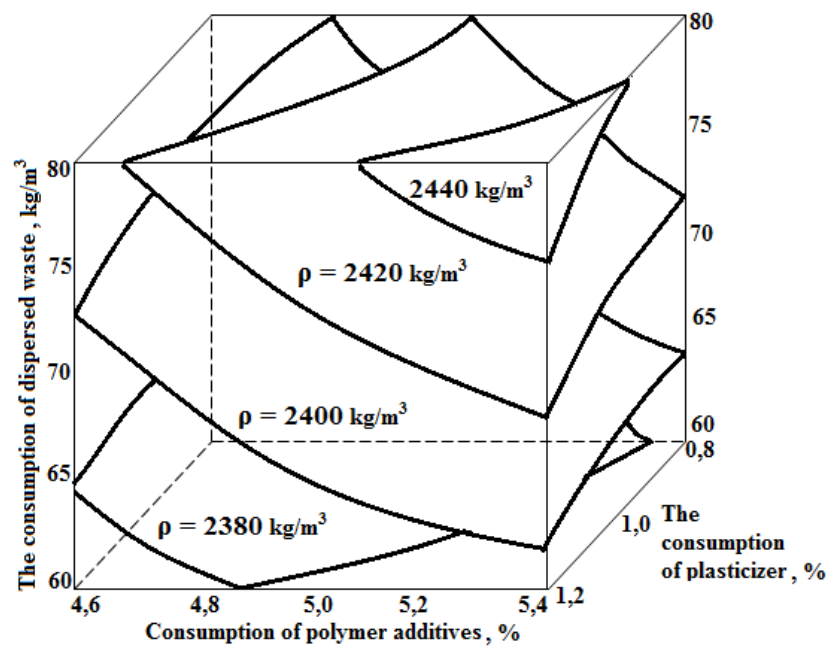

Fig. 2. Relation of the average density of polymer concrete samples (size 100x100x100 mm) on variable factors: dispersion waste costs, plasticizer and polymer additive.

\section{Discussion}

The influence of plasticizer consumption on the average density of concrete has an extreme significance, which makes it advisable to use the method of analytical optimization [5]. To find the extremum (the value of $\mathrm{X}_{2}$ ) corresponding to the minimum density, we use the method of analytical optimization.

The method of analytical optimization is based on a concept based on two statements: a mathematical model (in the form of a polynomial) is reasonable to the real process. It describes this process with an established degree of accuracy; and the resulting mathematical model is an algebraic nonlinear function of several variables: with this function, all types of actions can be performed using the apparatus of mathematical analysis [11-14]. The reasonable of the model is confirmed by the verification of statistical hypotheses, which is carried out in the process of processing the experimental data, the reasonable is determined by the Fisher criterion.

From the standpoint of mathematical analysis, analytical optimization consists in determining the extrema of a function of several variables for each of the variables (for this, find the partial derivatives for each of the variables (for each factor) and equate them to zero). Next, the polynomials are solved taking into account the found extremal functions and the optimized regression equations are obtained.

The analytical optimization technique is developed by NRU MGSU and tested in the study of technologies of various building materials and in the implementation of system solutions based on these materials.

When developing the methodology for selecting the composition of polymer concrete, optimization is carried out according to (plasticizer consumption), since only from this parameter does the average density have a quadratic functional dependence:

$$
\partial Y_{2} / \partial X_{2}=-14+16 X_{2}=0 \rightarrow X_{2}=\frac{14}{16}=0.875
$$

Having solved the equations $\mathrm{Y}_{1}\left(\mathrm{X}_{1}, \mathrm{X}_{2}, \mathrm{X}_{3}\right)$ and $\mathrm{Y}_{2}\left(\mathrm{X}_{1}, \mathrm{X}_{2}, \mathrm{X}_{3}\right)$ with $\mathrm{X}_{2}=0.875$, we obtain the following optimization functions:

- for compressive strength: 
- for medium density:

$$
Y_{1}=34.3+2.8 X_{1}+1.8 X_{3}-1.4 X_{1} X_{3}
$$

$$
Y_{2}=2409+21 X_{1}+12 X_{3}+10 X_{1} X_{3}
$$

We carry out a graphical interpretation of the dependence of compressive strength on the consumption of finely ground waste $\left(\mathrm{X}_{1}\right)$ and the consumption of the polymer additive $\left(\mathrm{X}_{3}\right)$. As a result, we obtain a nomogram (Fig. 3), with the help of which it is possible to predict the strength of polymer concrete or to choose the optimal costs of the components.

Determine the optimal consumption of plasticizer $(\mathrm{P})$ in natural terms: $\mathrm{P}=1.0+$ $0.2 \times 0.875=1.15 \pm 0.2 \%$

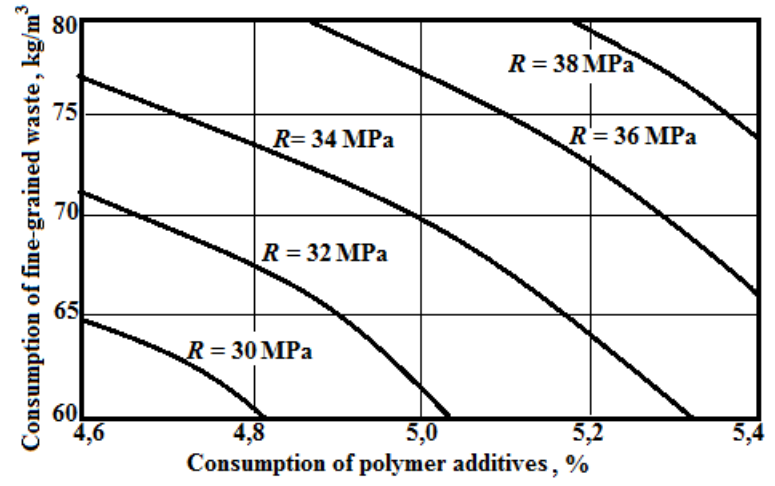

Fig. 3. Relation of concrete strength on the costs of fine milled waste and polymer additives with a plasticizer content of $1.15 \pm 0.2 \%$.

\section{Conclusion}

The methodology for selecting the composition and evaluating the properties of polymer concrete depending on the composition change was developed on the basis of mathematical planning of an active three-factor experiment, processing its results and using the methodology of analytical optimization. Cement consumption is $264 \mathrm{~kg} / \mathrm{m}^{3}$, sand consumption - $900 \mathrm{~kg} / \mathrm{m}^{3}$, coarse aggregate consumption $-1050 \mathrm{~kg} / \mathrm{m}^{3}$, water consumption $-210 \mathrm{dm}^{3} / \mathrm{m}^{3}$. To obtain polymer concrete with a strength not lower than $36 \mathrm{MPa}$, the waste consumption is assumed to be $66 \mathrm{~kg} / \mathrm{m}^{3}$; consumption of polymer additives $-5,1 \%$; optimized plasticizer consumption $-1,15 \%$.

After receiving the calculated values, their experimental verification is mandatory on the basis of test batches and tests at the age of 28 days with the necessary composition correction.

\section{References}

1. Telichenko V I, Oreshkin D V. Material science aspects of geoecological and ecological safety in construction //Ecology of urbanized areas. 2015. \#. 2. - P. 31-33.

2. Zhuk P M, Zhukov A D. The regulatory legal framework for the environmental assessment of building materials: prospects for improvement // Ecology and Industry of Russia. 2018. T.22, №4, P. 52-57

3. Pilipenko A S, Efimenko A Z. The use of construction waste from the demolition of panel buildings for the production of colored concrete. Vestnik MGSU. Special issue.\# 2008. P. 525-527

4. Pyataev E R, Pilipenko A S, Burtseva M A, Mednikova E A, Zhukov A D. Composite material based on recycled concrete FORM 2018. IOP Conf. Series: Materials Science and Engineering 032015(2018) doi:10.1088/1757-899X/365/3/032041 
5. Rumyantsev B M, Zhukov A D, Chkunin A S, Aristov D I. Optimization of cellular structures / Scientific Review \#. 13. P. 128-131.

6. Pyataev E, Medvedev A, Poserenin A, Burtseva M, Mednikova E and Mukhametzyanov $\mathrm{V}$, Theoretical principles of creation of cellular concrete with the use of secondary raw materials and dispersed reinforcement. https://doi.org/10.1051/matecconf/201825101012

7. Sheinfeld A V. Organo-mineral modifiers as a factor of increasing the durability of reinforced concrete structures / / Concrete and reinforced concrete. 2014. № 3. P. 1621.

8. Kaprielov S S, Sheinfeld A V, Dondukov V G. Cements and additives for the production of high-strength concrete / / Construction Materials. 2017. № 11. P. 4-10.

9. Efimenko A Z, Pilipenko A S, Kolyadin A A. Facade ground tiles on the aggregate of construction waste. Concrete technology. \# 7-8. 2009. P. 31

10. Buryanov A F, Krivenko V V, Zhukov A D, Moiseenko K S, Asamatdinov M O. Imitation marble // Construction Materials. 2016. \# 4. P. 102-104

11. Zhukov A D, Bobrova E Yu, Zelenshchikov D B, Mustafaev R M, Khimich A O. Insulation systems and green sustainable construction // «Advanced Materials. Structures and Mechanical Engineering». Volumes 1025-1026 (2014). P. 1031-1034

12. Rumjancev B M, Zhukov A D. Principles of creating new construction materials / VolgGASU Internet-herald. 2012. \#. 3(23). Available at: www.vestnik.vgasu.ru.

13. Kornev M V, Makarov A V, Tikhomirova I N, Karpenko M A. Corrosion of silicate materials // Construction Materials. 2017. № 12. P. 38-40.

14. Kaprielov S S, Sheinfeld A V, Kardumyan G S, Chilin I A. About the selection of rosters of high-quality concretes with organic-mineral modifiers // Construction Materials. 2017. № 12. P. 58-63.

15. Collepardi M. The New Concrete. Italy: Grafiche Tintoretto, 2006. 421 p.

16. Efimenko A Z, Pilipenko A S. Obtaining decorative finishing materials for facade systems on aggregates from concrete waste. Vestnik MGSU. Special issue \#.2009. P. $100-103$

17. Bessonov I V. Gypsum materials of the newgeneration for decorating the facades of buildings / Increasing the effectiveness of producing and using gypsum materials and products: mat. Of the All-Russ. seminar. Moscow. RAASN. April 2002. P. 82-87.

18. Aitcin H.-C. High-Performance Concrete. London and New York: E \& FN. 1998. 598 p 